\title{
A Preliminary Study of Business Model for Evolvable Production Systems
}

\author{
Antonio Maffei \\ Production Engineering \\ Royal Institute of Technologies \\ Stockholm, Sweden \\ maffei@iip.kth.se
}

\author{
Mauro Onori \\ Production Engineering \\ Royal Institute of Technologies \\ Stockholm, Sweden \\ onori@iip.kth.se
}

\begin{abstract}
The aim of this paper is to define, through a survey of the current achievements within the Evolvable Paradigm universe, the potential target companies for the Evolvable Manufacturing Systems and to describe some features of a business model that fits the EPS structure and requirements. Therefore, the new, main, concerns surfacing by the introduction of the Evolvable Paradigm into the industrial field have been underlined and analysed in order to put into evidence the economical advantages achievable with their adoption. A simplified version of the EPS methodology is hereby presented as a framework in which concept like life cycle of a product, investment evaluation tools and "coopetition" have been integrated enabling crossed considerations.
\end{abstract}

Keywords: Evolvable System, Business Model, Adaptive Control, Coopetition.

\section{INTRODUCTION AND LIMITATIONS}

A thoughtful analysis of the current scenario in manufacturing field has been performed through many roadmaps in recent years. ManuFuture [1], FutMan [2], Eupass [3] all agree that there is a strong need for sustainable and agile solutions to cope with the uncertainty linked to current technical and economical production issues. In other words the future manufacturing systems will have to be rapidly deployable and easily reconfigurable for new production. Current manufacturing, and especially assembly systems, unfortunately, do not fit this requirements because their development is mainly driven by the product they will realise [4], and their claimed "flexibility" refers often to volumes and batch changes. One of the answers to the needs listed above comes from the Evolvable Paradigm (Onori [5], Barata e al [6]) which has, since his appearance, been declined in many different fields. Major efforts in this direction has been done during the EUPASS project (included in the European Community $6^{\text {th }}$ framework programme) where a common architecture [7] along with a system setting methodology [4] have been developed and where an Ontolology for the representation of domain knowledge [8] and agentified control solutions [9] have been provided. As it is better detailed in the next paragraphs all these achievements lead to an innovative concept of manufacturing system which hardly fits in the traditional, rigid and well established way to approach the business nowadays. Therefore one of the purposes of this paper is, after an analysis of the Evolvable Paradigm's state of the art, to delineate some of the features of a business model that meets the requirements of an EMS.

Due to the broadness of the examined domain it is here necessary to introduce some limitations in order to avoid losing the focus and the effectiveness of the analysis. The realisation of a product involves a lot of different companies: beside the simple manufacturer (hence "the Company"), there are suppliers, distributors, and many other entities related to the manufacturing system's hardware and software settlement or to legal, financial and economical issues. Since this analysis is firstly aimed to underline the new needs related with the implementation at industrial level of an EPS consequently the attention is to be point on the entities directly in touch with the actual system. For this reason in this paper the studied object is the combined business models of the Company, the System Integrator (hence "SI") and the Module Supplier (hence the "MS") as it is considered both the core and the driver for future developments in the matter. It is, in facts, possible to assume that the other stakeholders while they will benefit of such a System, they will not needs major re-engineering of their assets and procedures as the ones above.

It is now useful, for a better understanding of the future paragraphs, a brief description, from the qualitative point of view, of the average present business approach of the entities listed above. The case about to be shown is quite typical and it is very useful not only to give an inside view on the matter, but also to set the boundaries and the hypothesis for this analysis. In the usual situation the Company, after the realisation of the full design of a new product, contacts the SI asking automatic solution to enable the manufacturing. Therefore, the SI, considering the already available assets buys new hardware from one or more MSs and it proceeds with the necessary physical and logical integration. It is possible then, that the common purposes push the players to join the efforts, for example a SI might ask for a module with customized layout, instead of the ones available on the MS's catalogue, or still it can suggest to the Company small adjustments on the product design to simplify some 
manufacturing task. In general we have many possible potential situations depending on several factors such as bargain power of the entities, volumes, nature of the product, technologies etc.

In such an approach further collaborations, beside the simple business relations (see fig 1), are occasional and not well structured: while, as it will be shown later, the intrinsic mechanisms created by the application of the Evolvable Paradigm lead to a natural cooperation between those entities.

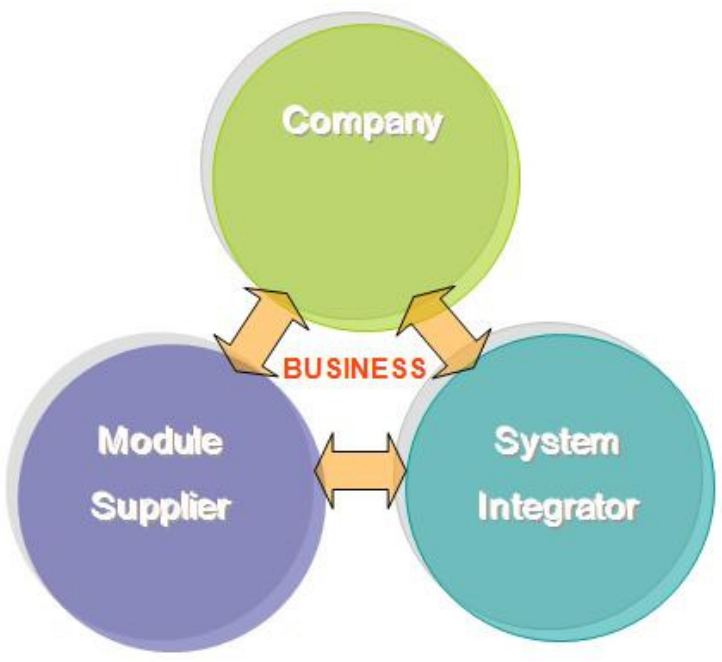

Fig. 1. Business-driven relations between the main entities involved in the realization of a manufacturing system

Setting and running a manufacturing system is a unique problem issue (requiring a holistic approach) and the simple figure above shows how, in the current model, there are three separated entities carrying it out. In such a model, we can assume that each player will rationally pursuit his local optimum and this of course will generate tradeoffs which are likely to drive the system equilibrium far from the global optimum. It is at this point clear that in order to achieve better results the entities above have to cooperate, and one of the aims of this paper is to shed light on the required relationships. To enable this Integrated Business Model it is necessary not only to have a common understanding of the matter, provided in this domain by a Reference Architecture [7], but also clear and accepted standards. This unification work has been one of the most important achievements in the Eupass Project [10].

It is now worth to remember that the adoption of an "intelligent" manufacturing system is often something that SMEs can't simply afford by themselves. A possible way to reduce the costs, exploiting both the Evolvable Paradigm and the "coopetition" strategy is presented later in the discussion. In the end one more consideration about the adoption of an "intelligent" system over a cheaper one: in the short term, the latter is more convenient while, as it is shown in the next paragraphs, the economical advantages of the first one surface only through years, different generations of a product and different projects. The point is that very often the CEOs are enrolled with few years' contracts, and they are paid mainly with stock options; therefore they will rather choose cheaper systems and outsourcing solutions to boost the shares than go for a long term strategy involving some expensive manufacturing system.

\section{METHODS}

In order to identify the specific requirements to approach business according with the Evolvable Paradigm, it is now useful to analyze some of his technical aspects. The main feature of an Evolvable System in general is his capability to adapt to new conditions and this means, in the manufacturing environment new products, new technologies, new volumes. While in the past the product was considered the basic issue for the company's success, with the evolvable paradigm the idea of using the same system for different productions and through more generations of a product pulls the production system itself as core entity [5]. An EMS is first of all a modular system, but not a traditional one: the modules are simple, task-specific and process-oriented. The processes are the driver of the modularization because they are the common denominator among different applications. The basic idea is that combining many modules, each with a specific atomic skill, it is possible not only to obtain a manufacturing system with the needed requirement but also to exploit new and unexpected capabilities surfaced from such coalition [6]: this is called Emergent Behavior. Controlling such set of modules entails a multi-agent approach [11], able to interpret the arising complexity. The basic skills belong to single module as well as the complex, composite skills reside in coalitions of modules. A process needs some skills to be done and therefore the skill becomes the link between processes and modules and in general between the product requirements and the actual production system [4]. The first challenge has been therefore to represent the basic processes in a structured and unambiguous way especially in those fields, such as the assembly, where there is no well defined process specification. For this purpose, starting from the actual user requirement specifications (overall project requirements and product model) a Process-oriented ontology has been developed within the EPS domain. This ontology has the aims to describe and guide the assembly process specification both for new assembly system configuration and for reconfiguration of existing assembly systems [8]. One of the most relevant consequences of this ontological representation is that the conceptual and physical links between the product's features and the actual manufacturing system will be evident since the first stages of a new project. In other words, having, during the development of the product, a clear picture of the system which will realize it, enables a feedback to the designers that is beyond simple DFM rules.

In order to fit in an EMS, the modules must have selfconsciousness and capability to communicate with other modules: embedded computational power and storage capacity are, as well as suitable interfaces and communication protocol, necessary requirements. Therefore, among the critical issues 
for the Evolvable Paradigm, the hardware standardization [10] and the development of agentified control solutions [12] have been deeply investigated. The actions within the modularization field are not only addressed to the realization of brand-new modules, but also in the adaptation and re-use of legacy equipment [13]. The discussed achievements picture an Evolvable Manufacturing System as a conceptual and physic platform featuring different modules, according with the specific requirement of the current application. The integration of such a system breaks the usual stepwise procedure (described in the introduction) in which each player has his task and defined moment of action. This is the main reason why current business models would fail in Evolvable Paradigm environment. One of the most interesting aspects of an Evolvable System is that it does not merely address to improve the equipment rate of reuse (which is actually already high enough) but according with the examined literature the application of such an approach should dramatically reduce some of the costs associated with: set-up, start-up, diagnosis, maintenance and reprogramming of the system [14] [15]. The proposed EAS methodology [4], specifically designed for an assembly system, but still valid for a whole manufacturing system, sheds light on the connections between the main entities involved in the EPS integration. Developing a simplified version of this methodology has been the first necessary step to attain consciousness of the problem. As it is shown in the next paragraph, the focus has been put on the bare actions required to run the system, while the most technical aspect have been neglected. The scheme is therefore the reference framework for the other elements surfaced during the analysis and it becomes the basis of subsequent discussion.

\section{RESULTS}

The Business Model of a company includes all the general details about the operations and decisions in its business: either from the strategic and from the technical point of view. Setting a business model entails, at first the definition of who the customer is and what the customer value. From that basic information, coming from marketing activities, is then possible to gather a general strategy, which then has to be detailed in all the different aspects of the business, from the supply to the production, from the maintenance to the distribution, from the financial aspects to the customer care. In other words, it is possible to consider the Business Model as the way the company uses to face external threats and to exploit opportunities. For this reason it is possible to consider the way a company develop his products and then builds them as the "core" of the Business Model.

The analysis performed in the previous paragraph revealed the needed concurrent approach in developing the product and the manufacturing system. The following figure is a simplification of the EPS methodology [4] and it summarizes the salient and relevant processes which allow the integration of an EMS.

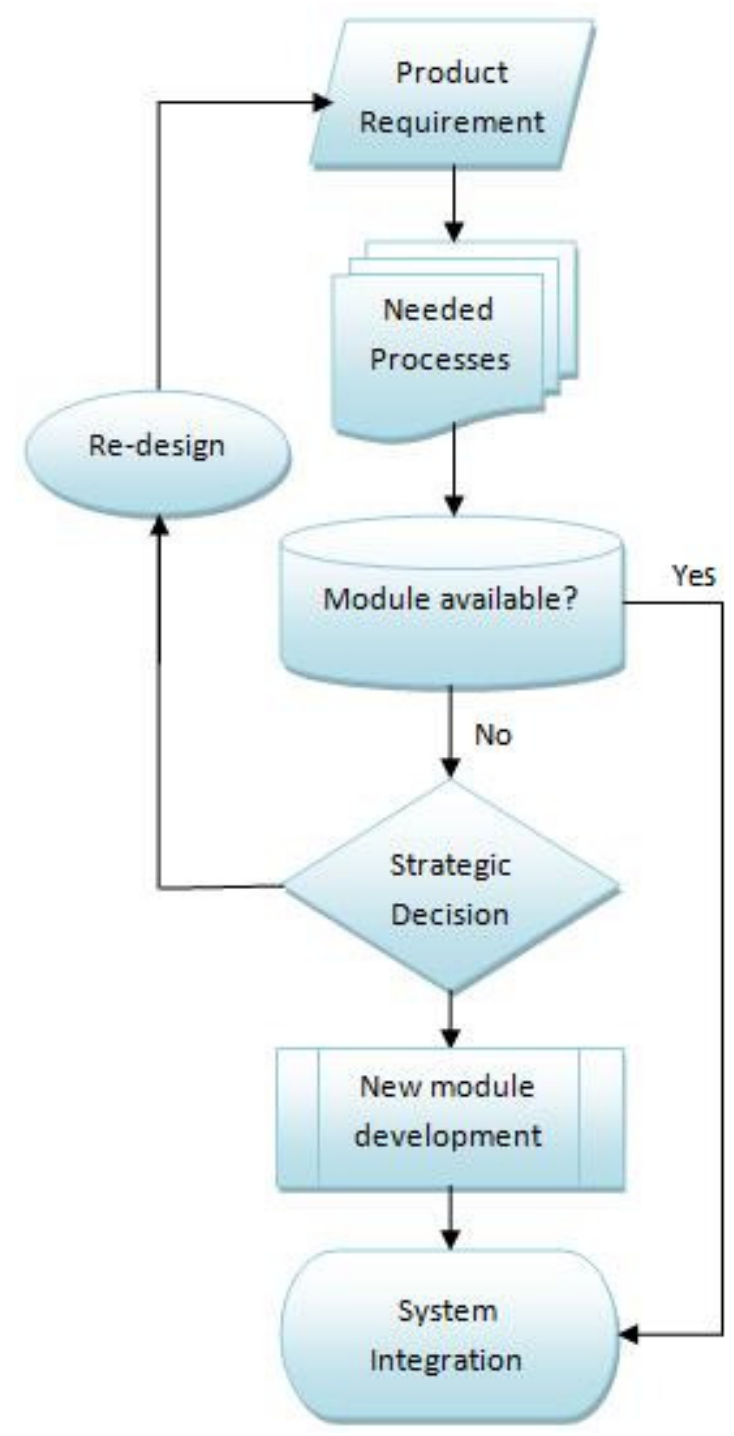

Fig.2. Simplified EPS Methodology

As this scheme underlines, in the EPS approach, the final product's features are not designed independently by the Company but they depend heavily on the feedbacks from the System Integrator and the Module Supplier. In fact, one can assume that such a scheme has two outputs, which at the end should be produced at the same time the Product Design and the Production System Design. The methodology applied within the EP is then based on a well structured, and multiplayer, concurrent engineering. The need of a rigorous ontological process description becomes here fundamental because the involved entities cooperate mainly through process information. The product is described as a set of processes, the choice of the module by the SI is made accounting of those processes, and new module from the MS must accomplish the exact needed process. In EMS environment a new project will need a minimum initial investment to reconfigure the layout of the existing assets (platform and modules) and a major investment in new 
modules to perform the task that the new product does not share with previous applications.

In the traditional business approach the initial investment for the manufacturing system is accounted on the budget of the single project which requires such a system. If then, the system is somehow flexible or re-usable is often possible though a re-engineerisation process to extend his lifespan to more projects. The investment on such a system is then likely to be spread on several products. From the cost accounting point of view is highly problematic to deal with this kind of system. Some of the questions that can here arise are: how much of the current cost are actually to be assigned to the present project and how much to the future ones? How much will cost to adapt the current system for the next application? Companies needs answer to these questions to set important parameters as Time to Market or Price, and to have accurate measure of their performances (ROI, NPV...). In the traditional manufacturing tasks, such as milling, drilling or turning it is rather easy to correctly account of the real costs associated with the processes. It is normal for companies to use machine tools over several projects (even at the same time), and the costing activities have to deal with the time of use and the set-up and support expenses. This is basically possible because the processes behind those activities are clear and very well defined. When it comes to less traditional tasks such as automatic assembly, where the processes are fuzzier, and the current solutions are more and less customized it is impossible to answer to the questions presented above.

The EMSs are built focusing on the manufacturing processes they will perform: for this reason they will be naturally used on different projects and through more generations of one product. In this sense they will enable costing strategies similar to the ones used for more traditional manufacturing activities, in more problematic contests also. Nevertheless, as it is shown below, the Evolvable Paradigm, exploiting the emergent behavior, allows considerations on the initial investment beyond mere re-usability of the components. The main feature of an Evolvable System is his capability to profit from past experience: each new module, each new solution allows the system to collect data and skills that can be profited in future applications. In this sense any investment on the system has the effect to broaden its skills and make it more valuable, and then less expensive, also for further projects. Of course it is necessary here to say that a modular system will never reach the performance in terms of volume and effectiveness of an integrated, purpose-made, one. The suitable environment for such a system is small to medium volumes in demanding and turbulent markets, but as it will be shown in the discussion they can be successfully applied also in more stable situations. Many companies are forced to go automatic, because of the nature of their products (microproducts, hazardous substances...) therefore "intelligent" evolvable manufacturing systems becomes a necessary tool to gain competitive advantage.

\section{DISCUSSION AND CONCLUSION}

The results of the analysis performed in this paper all lead to a need of a superior level of integration between the entities involved in the realization of an automated production system. The business model of each actor must include some areas in which the activities are done in strict cooperation with the others in order to achieve a global optimum. Referring to the scheme showed in the introduction the ideal overall situation can be pictured as below.

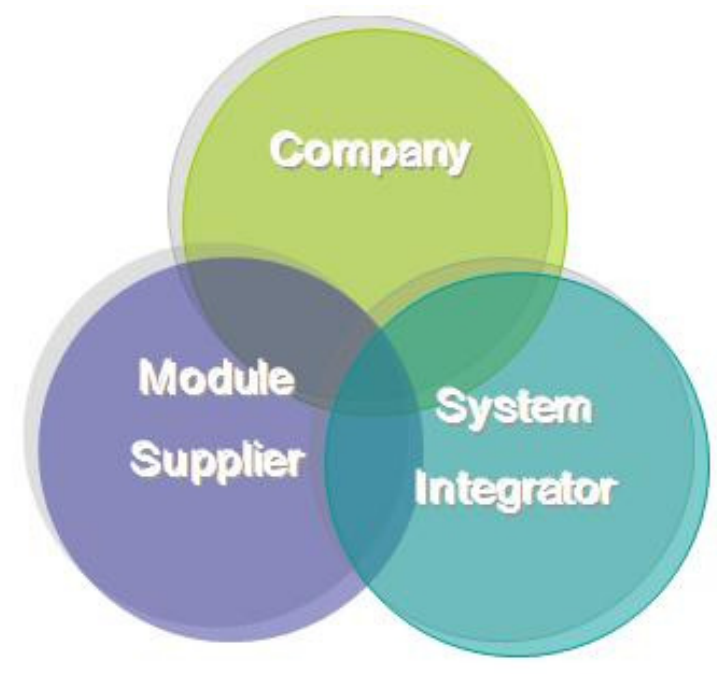

Fig.3 Integrated Business Model schematization

The intersections in this picture represent the activities described in the figure 2 . The main obstacle to this scheme is the lack of unique language, and in this sense the Evolvable Paradigm individuated the common denominator in the processes. Once the processes are well defined it is possible to refer them to a product, to a single module and then to a system. Traditional processes like milling have been structured and detailed over the years, and today it is possible and easy starting from a product to say what surfaces should be realized by milling, with what kind of tool and machine tool and where those operations should be placed within the working cycle. Once all this aspect will be clarified also for other, less traditional and structured, manufacturing processes it will be possible to enhance the autonomy of the system and to enable real evolution.

Another interesting aspect of the EPS is the modules management. The Company owns a physic platform where to implement the production system with the needed module. In this scenario there are no reasons, beside prospective strategic ones, to own more modules than the ones used in current applications. It is possible in other words to share the financial burden of storing modules with other companies that have the same platform in a "coopetition" logic. This common repository not only maximizes the rate of utilization of the existing modules but it also stimulates companies to join their $\mathrm{R} \& \mathrm{D}$ activities towards the creation of new ones. 


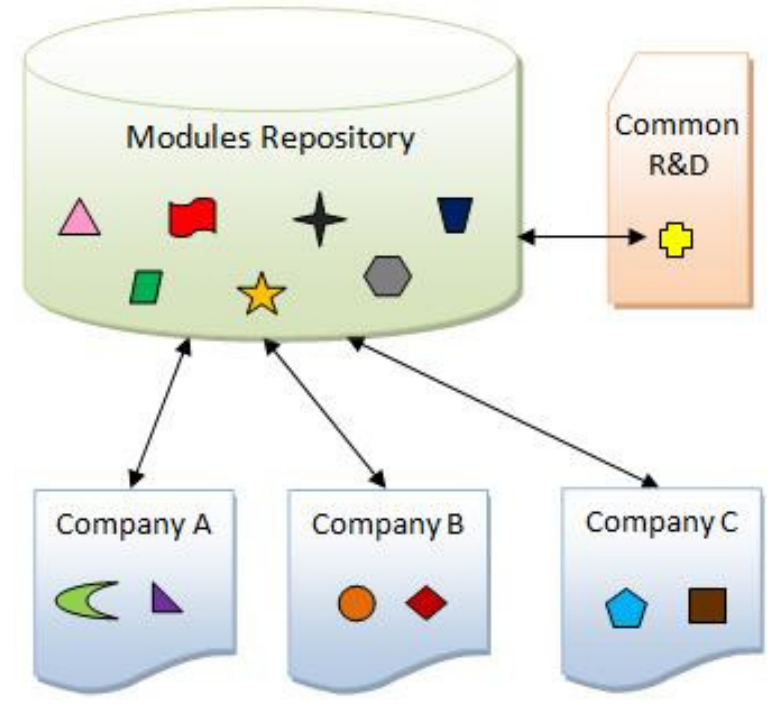

Fig.4 "Coopetition" in modules management

The Evolvable Paradigm has been created to help SMEs to have an automatic manufacturing system able to be economically convenient for small productions and for a wide range of product and applications but it could be useful also as a pre-production system able to assist a company in the passage from the launch of a new product to his maturity. Using an EMS to realize a product in the first stage of his lifecycle gives the option to build an expensive dedicated line only if it is justified by the sales volumes.

\section{Product Life Cycle}
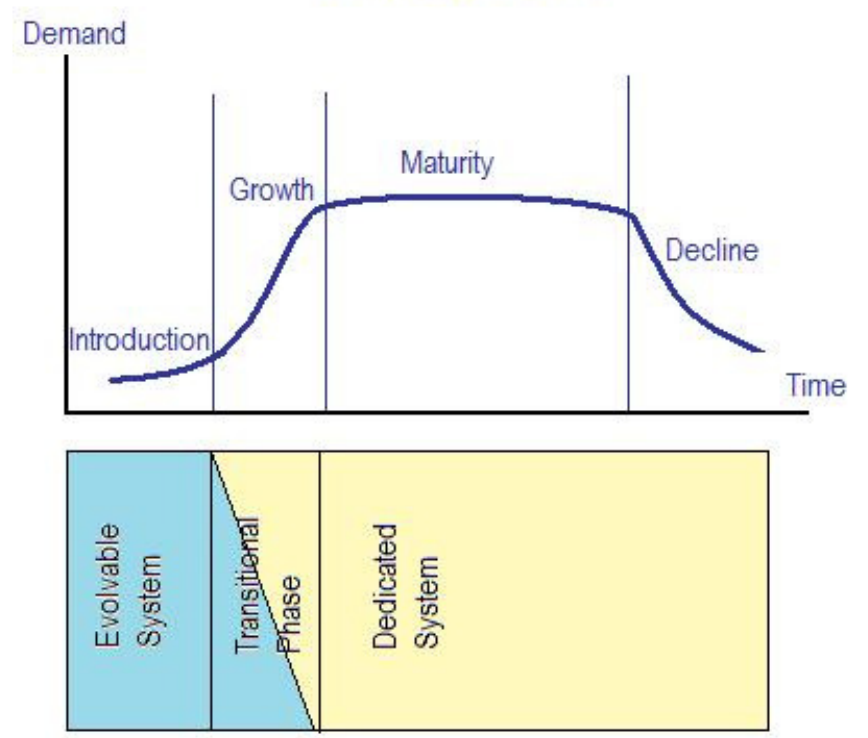

Fig.5 Different Manufacturing System for different phases of the lifecycle of a product

After the complete transition the EMS can be used for new purposes as well as backup system for the dedicated line.
The analysis performed hereby opens a lot of possible areas of investigation. Understanding that a higher degree of integration between the main actors is needed, it is now interesting to probe other business areas which have already faced successfully this kind of challenge. One of the most suitable sectors is for sure the IT. Many of the solutions suggested in this paper are in fact already applied successfully in our laptop. If you want to change a printer, an optical disc reader or even a CPU, it entails often few minutes. This is due to the use of the same physical interfaces, and to the control approach (using multi-agent).

The presented solutions need also to be corroborated from an economical point of view. For this reason, the current achievements in the field have to be evaluated with industrial test cases. An interesting application of the process-oriented control approach has been implemented by Introsys (a Portuguese company active in system integration [16]) in the automotive sector. A correct evaluation of the benefits attainable by EPS approach needs also the development of suitable accounting tools as well as new investment evaluation scheme.

In the end, in order to achieve real results in such a concept turn is necessary to disseminate the information and reach in this way the required critical mass. For this reason during the six months extension of the Eupass project, a network has been launched. In this website http://www.eas-env.org/ [17] is possible to find all the current achievement within the Evolvable Paradigm, and share experience, needs and solutions with a growing community.

\section{REFERENCES}

[1] -"Strategic Research Agenda-assuring the future of manufacturing in Europe"; Manufuture Platform-Executive Summary, Dec. 2005, EC

[2] -"The Future of Manufacturing in Europe 2015-2020-The Challenge for Sustaninability"; FutMan, Institute for Prospective Technological Studies, European Commission Joint Research Centre, EUR 20705 EN

[3] M.Onori, A.Maffei, J.Barata, C.Hanisch, T.Maraldo; "EUPASS- Adaptive Assembly Roadmap 2015"; Doc.D1.5f, EUPASS-Evolvable Ultra Precision Assembly, NMP-2-CT2004-507978; May 2008

[4] -Maffei A, Rossi T (in press 2007) "Development of an ontology to support Evolvable Assembly Systems: Electrolux test case", Master Thesis, KTH, Stockholm

[5] Onori, M. (2002a) In ISR2002 - 33rd International Symposium on RoboticsStockholm, pp. 617-621.

[6] Barata, J., Onori, M. and Frei, R. (2006) In ISIE'06 - IEEE International Symposium on Industrial ElectronicsIEEE, Montreal Canada. 
[7] M.van Zutphen,M.Onori, J.Barata; "EUPASS Architecture";

Document D4.10b, Project Report-Public, EUPASS-Evolvable Ultra

Precision Assembly, NMP-2-CT-2004-507978; March 2006

[8] Lohse N., Hirani H., Ratchev S., Turitto M.; “An Ontology for the definition and validation of assembly processes for Evolvable Assembly Systems"

[9] Barata,J., Onori,M., Frei,R., Leitão, P. (2007); "Evolvable Production Systems - Enabling Research Domains"; Proceedings of the 2nd International Conference on Changeable, Agile, Reconfigurable and Virtual Production (CARV 2007), Toronto, Canada on 22-24 July 2007

[10] -Hofmann,A., Siltala,N.(2009); "Emplacement and Blue Print An Approach to Handle and Describe Modules for

Evolvable Assembly Systems"; In 9th International IFAC

Symposium on Robot Control (SYROCO2009).

Gifu, Japan 9-12 September 2009

[11] Barata J.; "coalition Based Approach for Shop Floor Agility- a Multi-Agent approach" PhD thesis, Universida Nova de Lisboa, January 2004

[12] "Barata J, Ribeiro L, Onori M. ; Diagnosis on Evolvable Production Systems.”; Proc ISIE'07 - IEEE International Symposium on Industrial Electronics Vigo - Spain, 4-7 June, 2007.

[13] Adamietz, R.; "Development of an Intermodular Receptacle- A First Step in Creating EAS Modules"; MSC Thesis, KTH, Stockholm, Sweden, 2007.

[14] Maffei A., Dencker K., Bjelkemyr M., Onori M., (in press) "from Flexibility to Evolvability: Ways to Achieve Self-

Reconfigurability and Full Autonomy", In 9th International IFAC Symposium on Robot Control (SYROCO2009).Gifu, Japan 9-12 September 2009

[15] Onori, M., Barata, J. (in press). "Evolvable Production Systems : Mechatronic Production Equipment With Process-Based Distributed Control. In 9th International IFAC Symposium on Robot Control (SYROCO2009).

Gifu, Japan 9-12 September 2009

\section{[16] www.introsys.net}

\section{[17] http://www.eas-env.org/}

